\title{
Synergistic suppression of noscapine and conventional chemotherapeutics on human glioblastoma cell growth
}

\author{
Qi QI ${ }^{1}$, Xia LIU1 ${ }^{1}$, Shiyong $\mathrm{LI}^{1}$, Harish C JOSHI², Keqiang YE ${ }^{1, *}$ \\ ${ }^{1}$ Department of Pathology and Laboratory Medicine, ${ }^{2}$ Department of Cell Biology, Emory University School of Medicine, Atlanta, GA \\ 30322, USA
}

\begin{abstract}
Aim: Noscapine (NOS) is a non-narcotic opium alkaloid with anti-tumor activity. The aim of this study was to investigate the effects of the combination of NOS with conventional chemotherapeutics temozolamide (TMZ), bis-chloroethylnitrosourea (BCNU), or cisplatin (CIS)on human glioblastoma cells.

Methods: U87MG human glioblastoma cells were examined. Cell proliferation was quantified using MTT assay. Western blotting and flow cytometry were used to examine apoptosis and the expression of active caspase-3 and cleaved PARP. Mouse tumor xenograft model bearing U87MG cells was treated with TMZ (2 mg.kg-1 $\cdot \mathrm{d}^{-1}$, ip) or $\mathrm{CIS}(2 \mathrm{mg} / \mathrm{kg}$, ip 3 times a week) alone or in combination with NOS (200 mg. kg $\cdot \mathrm{d}^{-1}$, ig) for 3 weeks. Immunohistochemistry was used to investigate the expression of active caspase-3 and Ki67 following treatment in vivo. The safety of the combined treatments was evaluated based on the body weight and histological studies of the animal's organs.

Results: NOS (10 or 20 mol/L) markedly increased the anti-proliferation effects of TMZ, BCNU, and CIS on U87MG cells in vitro. The calculated combination index $(\mathrm{Cl})$ values of NOS-CIS, NOS-TMZ, and NOS-BCNU (20 $\mu \mathrm{mol} / \mathrm{L})$ were $0.45,0.51$, and 0.57 , respectively, demonstrating synergistic inhibition of the drug combinations. In tumor xenograft models, combined treatment with NOS robustly augmented the anti-cancer actions of TMZ and CIS, and showed no detectable toxicity. The combined treatments significantly enhanced the apoptosis, the activated caspase-3 and PARP levels in U87MG cells in vitro, and reduced Ki67 staining and increased the activated caspase-3 level in the shrinking xenografts in vivo.

Conclusion: NOS synergistically potentiated the efficacy of FDA-approved anti-cancer drugs against human glioblastoma cells, thereby allowing them to be used at lower doses and hence minimizing their toxic side effects.
\end{abstract}

Keywords: glioblastoma; noscapine; temozolamide; bis-chloroethylnitrosourea; cisplatin; apoptosis; caspase-3; tumor xenograft model; drug synergism

Acta Pharmacologica Sinica (2013) 34: 930-938; doi: 10.1038/aps.2013.40; published online 27 May 2013

\section{Introduction}

Noscapine (NOS), a naturally occurring anti-tussive phthalideisoquinoline alkaloid, is a tubulin-binding, anti-cancer agent. It binds to tubulin, altering its conformation and assembly properties $^{[1]}$ and interfering with microtubule dynamics ${ }^{[2,3]}$. However, NOS does not significantly change the microtubule polymer mass, even at higher concentrations ${ }^{[2,4]}$. NOS arrests the cell cycle in a variety of mammalian cells, including drugresistant variants, targeting these cells for apoptosis ${ }^{[1,4-6]}$. It also inhibits the progression of many cancer types and has no detectable toxicity to rapidly dividing cells and post-mitotic

\footnotetext{
* To whom correspondence should be addressed.

E-mail kye@emory.edu

Received 2012-12-25 Accepted 2013-03-21
}

cells, such as neurons. Notably, NOS does not inhibit primary humoral or cellular immune responses in mice ${ }^{[7]}$.

Unlike currently available anti-microtubule drugs, such as taxanes and vincas, NOS is orally available, well-tolerated and shows no detectable toxicity in mice and humans ${ }^{[8,9]}$. Microtubules are major cytoskeletal structures that are responsible for maintaining genetic stability during the mitotic phase of the cell cycle ${ }^{[10]}$. The highly dynamic nature of microtubules is required for mitosis ${ }^{[1]]}$. Microtubule inhibitors are classified into two groups: stabilizing and destabilizing agents. Microtubule-stabilizing agents, including taxanes and epothilones, operate by promoting polymerization and increasing the microtubule polymer mass in cells. Destabilizing agents, such as the vinca alkaloids, depolymerize microtubules, inhibit polymerization, and decrease the polymer mass ${ }^{[12]}$. The effec- 
tiveness of microtubule-targeting drugs for cancer therapy is impaired by various side effects, notably neurological and hematological toxicities ${ }^{[11]}$. Anti-microtubule drugs that are currently used in the clinic, such as vincas and taxanes, have limited efficacy due to the emergence of drug resistance. Multiple mechanisms of anti-microtubule drug resistance have been described, including overexpression of drug-efflux pumps, altered expression of tubulin isotypes, and mutational lesions in tubulin itself ${ }^{[13-15]}$. It has also been shown that NOS and its analogs can be used to successfully treat paclitaxel resistant ovarian cancer and T-cell lymphoma ${ }^{[4,16]}$.

Gliomas are the most common primary tumors of the brain. Glioblastoma multiforme (GBM), grade IV, is the most frequent and aggressive glioma, with a median survival of approximately 12-14 months, despite complete surgical resection followed by radiation and chemotherapy with temozolamide (TMZ). The poor prognosis for GBM has not improved for several decades. Hence, novel and effective therapeutic interventions for GBM are urgently needed to increase the survival of GBM patients. TMZ is an oral alkylating agent that is used for the treatment of Grade IV GBM and melanoma. Another drug in this class of agents is carmustine, or bis-chloroethylnitrosourea (BCNU), a mustard gas-related $\beta$-chloronitrosourea compound that is able to form interstrand crosslinks in DNA, thereby preventing DNA replication and transcription. Cisplatin (CIS) is also a canonical drug for the treatment of malignant gliomas ${ }^{[17]}$. All of these drugs are cytotoxic DNA damaging agents that display unfavorable side effects in clinical application.

Because NOS crosses the blood-brain barrier and inhibits glioblastoma growth ${ }^{[6]}$, we tested whether it has a synergistic effect in combination with the three FDA-approved drugs mentioned above. Here, we report that the combination of NOS and TMZ, BCNU, or CIS increases the cytotoxicity of these standard chemotherapeutic agents toward U87MG glioblastoma cells, and these combination therapies have pronounced synergistic anti-tumor activities in vivo. These findings warrant further development of a combinatorial therapy regimen for treating a variety of human cancers, including glioblastoma.

\section{Materials and methods} Cells, reagents, and mice

U87MG human glioblastoma cells were cultured in DMEM supplemented with $10 \%$ FBS and penicillin (100 units/mL)streptomycin $(100 \mathrm{~g} / \mathrm{mL})$, and glutamine $(2 \mathrm{mmol} / \mathrm{L})$ and maintained at $37^{\circ} \mathrm{C}$ with $5 \% \mathrm{CO}_{2}$ in a humidified incubator. CIS, NOS, TMZ, 3-(4,5-dimethylthiazol-2-yl)-2,5-diphenyltetrazol-iumbromide (MTT), and BCNU were purchased from Sigma, USA. NOS, TMZ, CIS, and BCNU were freshly prepared in dimethyl sulfoxide (DMSO) as stock solutions at a concentration of $100 \mathrm{mmol} / \mathrm{L}$. Then, cells were treated with the drugs at the indicated concentrations (after dilution of the stock solution in culture medium). Active-caspase-3 and Ki67 antibodies were purchased from Cell Signaling, Inc (Danvers, MA, USA). The horseradish peroxidase linked IgG secondary antibody was purchased from GE healthcare (Pittsburg, PA, USA). The Histo-SP AEC kit was obtained from Invitrogen, Inc (Grand Island, NY, USA). Nude mice (nu/nu) (for tumor growth assay) and C57BL/6J mice (for toxicological revaluation) of 5-6 weeks of age were obtained from the NCI. The mice were housed in standard conditions ( 5 mice/cage maximum) and fed autoclaved chow and water, with $12 \mathrm{~h}$ light and dark cycles. All efforts were made to minimize the animals' discomfort. The animals required physical restraint for the injection of tumor cells, delivery of drugs, and measurement of tumor size with calipers (hand-held). All procedures were approved by the Institutional Animal Care and Use Committee of Emory University.

\section{In vitro proliferation assay and flow cytometric analysis}

Cells were seeded and cultured in 96-well plates (4000 cells/ well). The next day, the medium was replaced with fresh medium containing different concentrations of the drugs or vehicle controls. The cells were incubated at $37^{\circ} \mathrm{C}$ for the indicated times. After treatment, the cells were incubated for three hours with $0.5 \mathrm{mg} / \mathrm{mL}$ MTT solution at $37^{\circ} \mathrm{C}$. Finally, the solution was decanted, and $100 \mu \mathrm{L}$ of DMSO was added to dissolve the purple formazan crystals. The absorbance of the resulting solution was measured at $570 \mathrm{~nm}$ with a microplate reader (Synergy 2, BioTek, VT, USA). Flow cytometric analysis was used to quantitatively examine apoptosis after drug treatment. After $24 \mathrm{~h}$ of treatment, the cells were stained with propidium iodide and analyzed with flow cytometry, as described previously ${ }^{[18]}$.

\section{Determination of combination index}

The interaction between NOS and the chemotherapeutics was quantified by determining the combination index (CI), which was calculated according to the median-effect principle ${ }^{[19]}$. The equation for the isobologram was as follows: $C I=(D) 1 /$ (Dx)1+(D)2/(Dx)2, where (Dx)1 and (Dx)2 indicate the individual dose of NOS and the chemotherapeutic required to inhibit a given level of cell growth, and (D)1 and (D)2 indicate the doses of NOS and the chemotherapeutic necessary to produce the same effect in combination, respectively. The combined effects were scored on the following scale: $\mathrm{CI}<1$, synergism; CI=1, additive effect; and CI $>1$, antagonism. Data analysis was performed with Calcusyn software (Biosoft, Oxford, UK).

\section{Tumor xenograft studies}

U87MG cells $\left(4 \times 10^{6}\right)$ in $100 \mu \mathrm{L}$ of serum free DMEM medium were inoculated subcutaneously (sc) into one flank of 5- to 6-week-old, female nude mice. Treatment commenced once tumors reached the mean volume indicated in the appropriate figure legend. The mice were randomly divided into different groups that were treated with vehicle $(0.9 \% \mathrm{NaCl}$ solution, QD), TMZ (2 mg/ kg, ip QD), CIS (2 mg/ kg, ip 3 times a week), NOS (200 mg/kg, ig QD), TMZ (2 mg/kg, ip) with NOS (200 $\mathrm{mg} / \mathrm{kg}$, ig), or CIS (2 mg/kg, ip) with NOS (200 mg/ kg, ig) for three weeks. Tumor volume $\left(\mathrm{mm}^{3}\right)$ was determined using the 
formula (length $\times$ width $\left.{ }^{2}\right) / 2$, where length was the longest axis and width was the measurement perpendicular to the length. Data are expressed as mean tumor volume \pm SD for each treatment group. Twenty-four hours after the last treatment, the animals were sacrificed, and the tumors were collected and weighed.

\section{Immunohistochemistry analysis}

Tumors were fixed in $4 \%$ paraformaldehyde overnight, followed by paraffin embedding. Sections $(8 \mu \mathrm{m})$ were deparaffinized in xylene and rehydrated in graded alcohols. Endogenous peroxidase activity was blocked by $3 \%$ hydrogen peroxide for $5 \mathrm{~min}$, and all slides were boiled in $10 \mathrm{mmol} / \mathrm{L}$ citrate buffer ( $\mathrm{pH}$ 6.0) for $10 \mathrm{~min}$. Acitvie-caspase-3 and Ki67 were detected using specific primary antibodies and the Zymed Histo-SP AEC kit. The slides were then counterstained with hematoxylin.

\section{Western blot analysis}

Cells were lysed in RIPA buffer and centrifuged for $15 \mathrm{~min}$ at $4^{\circ} \mathrm{C}$. Total protein was quantified using the Bradford reagent, and equal amounts of total protein were mixed with $4 \times$ SDS sample buffer, incubated at $95^{\circ} \mathrm{C}$ for $5 \mathrm{~min}$, and separated by SDS-PAGE. After electrophoresis, proteins were transferred to a nitrocellulose filter membrane (Bio-Rad, MA, USA) and blocked for $1 \mathrm{~h}$ at room temperature. Each membrane was incubated with the appropriate primary antibody at $4{ }^{\circ} \mathrm{C}$ overnight. The blots were then incubated with HRP-conjugated secondary antibodies for $1 \mathrm{~h}$, washed three times with PBST, and visualized using the Immobilon Western Chemiluminescent HRP Substrate (Millipore Co, Billerica, MA, USA).

\section{Toxicological study}

To assess changes in hepatic and renal function after treatment, blood was collected by heart puncture using heparinrinsed $1 \mathrm{~mL}$ syringes prior to sacrifice. The levels blood urea nitrogen (BUN) and creatinine (Cr) were used to assess changes in liver and renal function. For histological analyses, formalin-fixed, paraffin-embedded tissue sections were stained with H\&E and evaluated by light microscopy. The tissues examined included the heart, lung, brain, liver, kidney, spleen, muscle, small intestine, and stomach.

\section{Statistical analysis}

Data are presented as the mean \pm SD of two or three independent experiments. Statistical evaluation was performed using the Student's $t$-test or one-way ANOVA. Differences were considered to be statistically significant when $P<0.05$. All statistical analyses were performed using Prism software (GraphPad Software, La Jolla, CA, USA).

\section{Results}

Synergistic anti-proliferative effect of combined treatment with conventional chemotherapeutics and NOS

As NOS exhibited tumor-inhibitory activity in a pre-clinical model of $\mathrm{GBM}^{[6,20]}$, we further explored whether NOS demon- strates a therapeutically beneficial effect when administered in combination with canonical chemotherapeutics. U87MG cells were treated with 10 or $20 \mu \mathrm{mol} / \mathrm{L}$ of NOS in the presence or absence of the indicated drugs at different doses $(5,10,20,50$, and $100 \mu \mathrm{mol} / \mathrm{L})$ for $72 \mathrm{~h}$. Cell proliferation assays revealed that TMZ alone suppressed proliferation in a dose-dependent manner. When combined with NOS, TMZ displayed much stronger anti-proliferative activity toward U87MG cells (Figure 1A). Similar effects were observed with BCNU and CIS (Figure $1 \mathrm{~B}$ and $1 \mathrm{C}$ ). NOS has been extensively studied, and it is considered to be a promising anti-cancer drug with low toxicity $^{[21]}$. We next examined whether these chemotherapeutics also exert a similar effect in the presence of NOS. Accordingly, we treated cells with 10 or $20 \mu \mathrm{mol} / \mathrm{L}$ of TMZ, BCNU or CIS in combination with different concentrations of NOS (5, $10,20,50$, or $100 \mu \mathrm{mol} / \mathrm{L})$. MTT assays revealed that the combination of NOS with TMZ, BCNU, or CIS enhanced the antiproliferative effect of NOS (Figure 1A, 1B, and 1C). Higher concentrations $(20 \mu \mathrm{mol} / \mathrm{L})$ of TMZ, BCNU, or CIS showed a much more potent effect than the lower concentration (10 $\mu \mathrm{mol} / \mathrm{L})$ when combined with NOS. To further confirm the observed synergistic effect, we employed Median Effect Analysis. The combined effect of NOS and TMZ/BCNU/CIS at 10 and $20 \mu \mathrm{mol} / \mathrm{L}$ (NOS: TMZ/BCNU/CIS molar ratio was 1:1) was determined using the combination index (CI) method of Chou and Talalay ${ }^{[18]}$. The calculated CI values $(20 \mu \mathrm{mol} / \mathrm{L})$ of NOS-CIS, NOS-TMZ, and NOS-BCNU were $0.45,0.51$, and 0.57 , respectively. The CI value for each combination was $<1$ (Figure 1D), indicating there is a synergistic inhibitory effect of these drug combinations on U87MG cells ${ }^{[22]}$. Collectively, our data show that NOS dramatically potentiates the anti-proliferative activities of TMZ, BCNU, and CIS against U87MG cells.

\section{Synergistic anti-tumor activities of the combined chemotherapies} in vivo

We next tested whether low doses of TMZ/CIS displayed enhanced anti-cancer activity when concomitantly delivered together with NOS in tumor xenograft models. Nude mice were subcutaneously injected with $4 \times 10^{6}$ U87MG cells in the dorsal flank region. Tumors were allowed to grow to approximately $100 \mathrm{~mm}^{3}$ in size. Then, the mice were randomized into 6 groups and treated either with one of the following regimens: vehicle alone, individual drugs alone (TMZ, NOS, CIS), or individual drugs in combinations with NOS (Figure 2A). TMZ and CIS were administered via intraperitoneal injection, whereas NOS was administered via oral gavage once a day for 21 consecutive days. The tumor growth curve demonstrated that NOS alone displayed a remarkable inhibitory effect, TMZ exhibited a lower anti-proliferative activity than NOS, and CIS showed modest activity. Nonetheless, the combination of TMZ and NOS potently suppressed tumor growth, whereas the combination of CIS and NOS slightly reduced the tumor suppressive activity (Figure 2A). Quantitative analysis of the tumor weights fit well with the tumor growth curve (Figure 2B). Compared to the mice that were treated with single drugs, NOS treatment significantly shrunk the tumor size. 

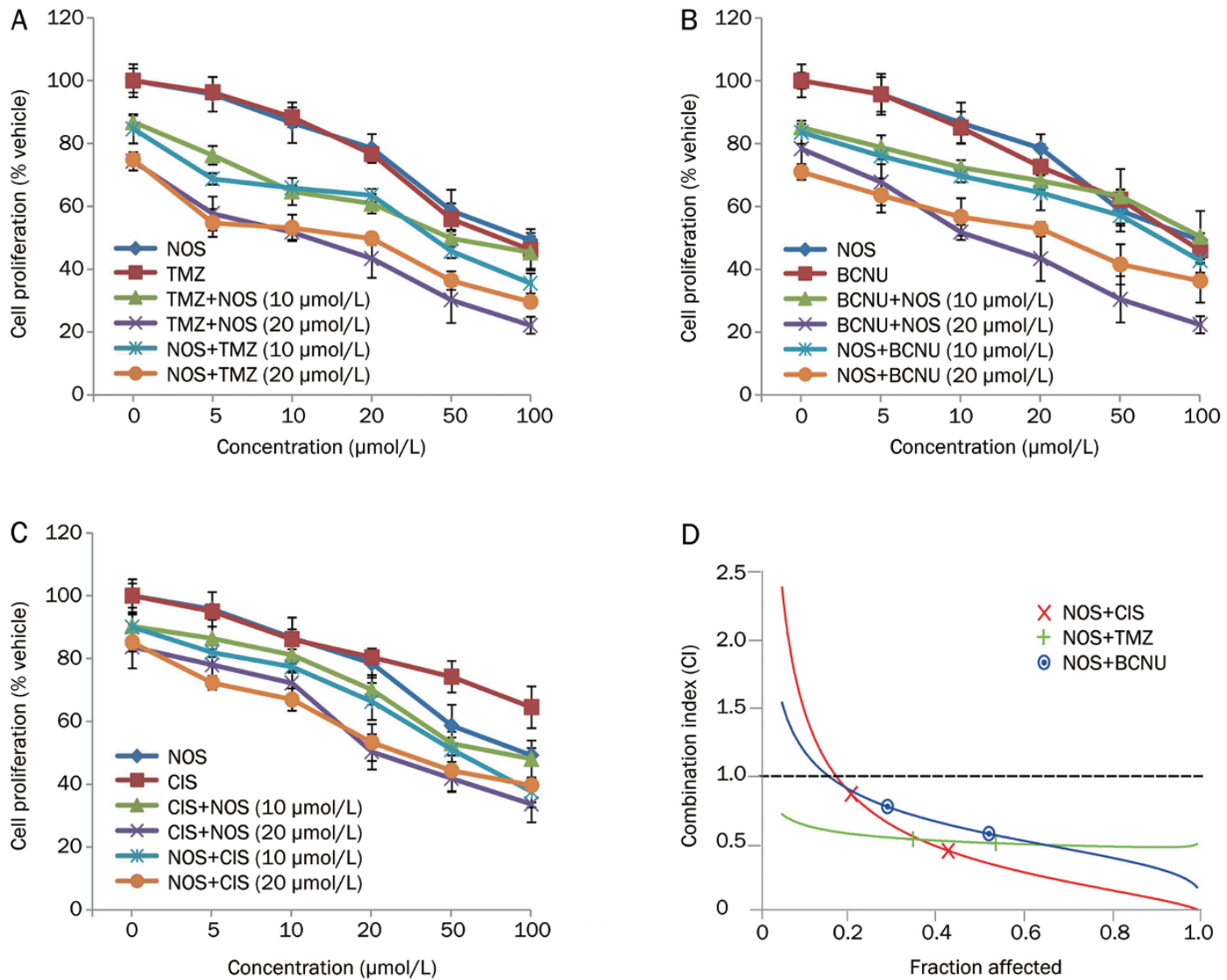

Figure 1. Synergistic effect of NOS and classical chemotherapeutics in U87MG glioblastoma cells. U87MG cells were seeded in 96-well plates (4000 cells/well) and treated with increasing or constant (10 or $20 \mu \mathrm{mol} / \mathrm{L}$ ) concentrations of NOS in combination with TMZ (A), BCNU (B), or CIS (C) for $72 \mathrm{~h}$. MTT assays were used to assess cell proliferation in each treatment group, as described in Materials and Methods. Data are expressed as the mean \pm SD. (D) Isobologram and combination index analyses of the inhibition of U87MG cell proliferation following treatment with the combination of NOS and different chemotherapeutics. When the fractions affected are higher than $0.2, \mathrm{Cl}<1$ indicates a synergistic effect. NOS, noscapine; TMZ, temozolamide; BCNU, bis-chloroethylnitrosourea; CIS, cisplatin.

The most impressive results were obtained with the combination of TMZ and NOS (1.87-fold decrease by tumor size and 2.05 -fold decrease by tumor weight), followed by the CISNOS group (1.72-fold decrease by tumor size and 1.66-fold decrease by tumor weight) (Figure $2 \mathrm{~B}$ and $2 \mathrm{C}$ ). The $\mathrm{CI}$ values were 0.471 and 0.695 for the TMZ-NOS and CIS-NOS treated groups, respectively. These data indicate that NOS, when given in a combinatorial regimen, significantly decreases the doses of TMZ and CIS required to achieve therapeutic efficacy in GBM treatment.

\section{Synergistic apoptosis-inducing effect of NOS and conventional chemotherapeutics on U87MG cells in vitro and in vivo}

NOS was previously shown to induce apoptosis in glioblastoma cells at a concentration of $100 \mu \mathrm{mol} / \mathrm{L}$ in vitro ${ }^{[23]}$. The concentrations needed for TMZ, BCNU, and CIS to exert their apoptosis-inducing effect are between 50 and $200 \mu \mathrm{mol} / \mathrm{L}^{[24-26]}$. Our data demonstrated that NOS significantly enhanced the anti-cancer effect of these chemotherapeutics in vitro and in vivo. To determine whether apoptosis was responsible for the tumor shrinkage observed in our experiments, we examined the levels of active caspase- 3 and PARP cleavage by using Western blotting. As shown in Figure 3A, TMZ, BCNU, CIS, and NOS alone moderately enhanced the expression of active caspase- 3 compared to the control. However, the combination of these chemotherapeutics and NOS notably increased the levels of active caspase-3 (Figure 3A and 3B). Moreover, co-treatment with NOS and TMZ, BCNU, or CIS induced PARP cleavage (Figure 4A). We further quantified the levels of apoptosis by flow cytometric analyses. As shown in Figure 3C, combination therapy with NOS synergistically enhanced the apoptosis-inducing effect of TMZ, BCNU, and CIS compared to treatment with the single agents alone. Immunohistochemical analysis showed that combination therapy with NOS and TMZ or CIS increased the active-caspase- 3 levels in tumor tissues, which was consistent with our in vitro data (Figure 3D). Moreover, Ki67 staining revealed that cell proliferation was significantly reduced by the combination therapies compared to single drug treatment (Figure 3D). 
A

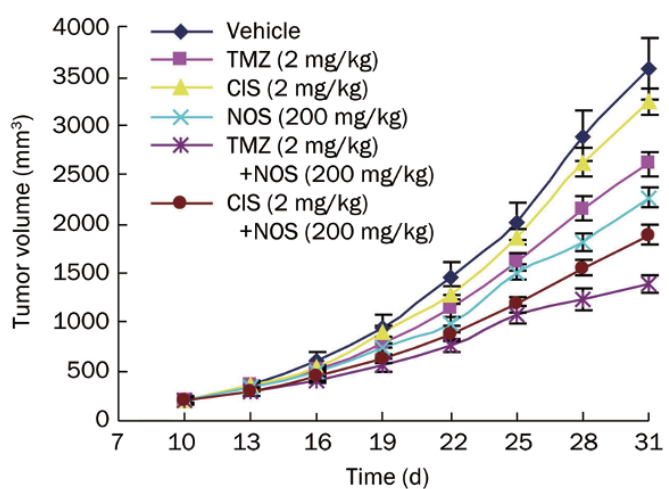

B

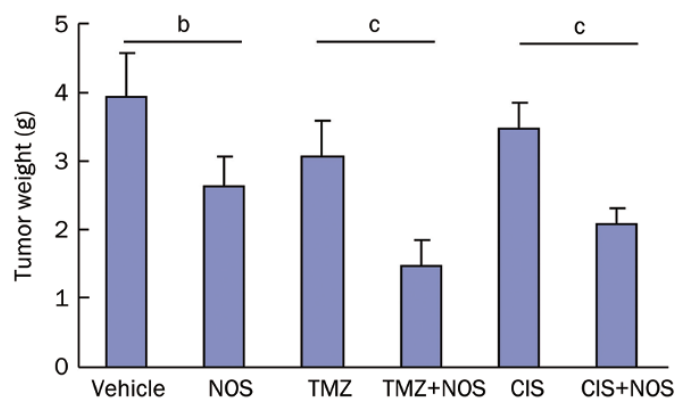

C

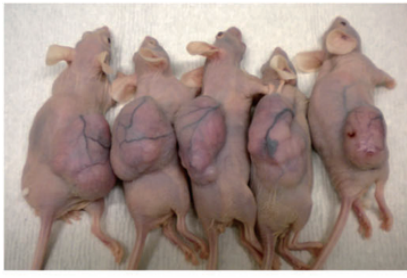

Vehicle

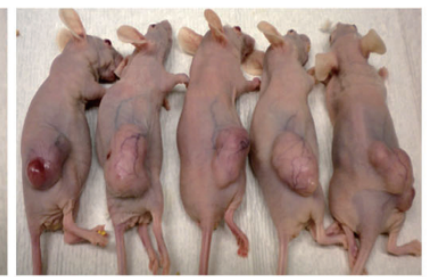

NOS

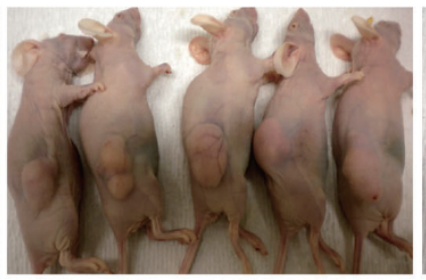

CIS +NOS

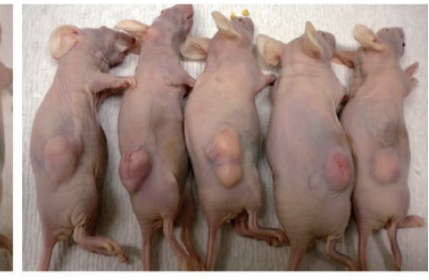

TMZ+NOS

Figure 2. Efficacy of NOS in combination with TMZ/Cisplatin in GBM xenograft model bearing U87MG cells. U87MG cells were inoculated subcutaneously in nude mice, tumors were allowed to form, and then mice were treated with drugs, as described in Materials and Methods. Tumor volumes (A) and tumor weight (B) were examined throughout the experiment. Data represent the mean \pm SD $\left({ }^{b} P<0.05,{ }^{c} P<0.01, n=5 /\right.$ group, two independent experiments). (C) Representative examples of the U87MG tumor bearing mice. NOS, noscapine; TMZ, temozolamide; CIS, cisplatin.

\section{Toxicological evaluation}

In clinical studies, the standard doses for TMZ and CIS are $150 \mathrm{mg} / \mathrm{m}^{2}$ (33.8 mg/ $\mathrm{kg}$ for mice) and $75 \mathrm{mg} / \mathrm{m}^{2}(16.9 \mathrm{mg} / \mathrm{kg}$ for mice), respectively. At these doses, these drugs have significant side effects. The most common side effect of TMZ treatment is gastrointestinal toxicity ${ }^{[27]}$, and the major reported toxicity from CIS treatment is nephrotoxicity ${ }^{[28]}$. Because therapy-limiting toxicities are a major impediment to effective chemotherapy, we performed toxicological evaluations using experimental animals. Mice were treated with the same drug combinations described previously, and two additional groups were added: high dose TMZ and CIS single agent treatments. Mice treated with the combination therapies showed no significant changes in body weight compared to the controls. In contrast, the body weights of animals treated with high doses of TMZ and CIS decreased rapidly over the duration of drug treatment (Figure 4A). Biochemical analyses of serum revealed abnormal kidney function with increased BUN and $\mathrm{Cr}$ in the mice treated with high doses of CIS or TMZ, whereas the combination of these drugs and NOS exerted no adverse effect on these parameters (Table 1). Hematoxylin and eosin staining revealed that the NOS and CIS/TMZ treated mice showed no demonstrable histological changes compared to the control mice (data not shown). However, high dosages of TMZ (33.8 mg/ $\mathrm{kg}$ ) and CIS $(8.4 \mathrm{mg} / \mathrm{kg}$ ) led to kidney damage (Figure 5B). Taken together, these data indicate that, compared to vehicle controls, the combination of low dose CIS/ TMZ and NOS exerted no detectable toxicity in vivo.
Table 1. Biochemical profiles of plasma of C57 mice treated with temozolamide (TMZ), cisplatin (CIS), or combined with noscapine (NOS).

\begin{tabular}{lcc}
\hline \multicolumn{1}{c}{ Group } & \multicolumn{2}{c}{ Biomedical enzymes } \\
& BUN (mg/DI) & $\mathrm{Cr}(\mathrm{mg} / \mathrm{dL})$ \\
\hline Vehicle & $28 \pm 2.83$ & $0.3 \pm 0.14$ \\
$\mathrm{TMZ}(2 \mathrm{mg} / \mathrm{kg})+\mathrm{NOS}(200 \mathrm{mg} / \mathrm{kg})$ & $19.5 \pm 6.36$ & $0.3 \pm 0.14$ \\
$\mathrm{TMZ}(33.8 \mathrm{mg} / \mathrm{kg})$ & $144 \pm 87.68^{\mathrm{b}}$ & $0.75 \pm 0.35^{\mathrm{b}}$ \\
$\mathrm{CIS}(2 \mathrm{mg} / \mathrm{kg})+\mathrm{NOS}(200 \mathrm{mg} / \mathrm{kg})$ & $25 \pm 7.07$ & $0.35 \pm 0.21$ \\
CIS $(8.4 \mathrm{mg} / \mathrm{kg})$ & $204 \pm 67.88^{\mathrm{b}}$ & $1.1 \pm 0.42^{\mathrm{b}}$ \\
Normal range & $8-33$ & $0.2-0.9$ \\
\hline
\end{tabular}

Biochemical parameters were measured by collecting the blood after the end of treatments. BUN, blood urea nitrogen; $\mathrm{Cr}$, creatinine. Data are shown by mean $\pm S D$, derived from 3-4 samples for each statistical analysis. ${ }^{\mathrm{b}} \mathrm{P}<0.05$ vs vehicle group.

\section{Discussion}

Cancer is driven by a combination of gene mutations. Accordingly, blockage of one specific signaling pathway using targeted monotherapy has negligible therapeutic efficacy in most human cancers. The combination of various chemotherapeutic agents is broadly used for treating a variety of cancers. Owing to the highly infiltrative and invasive features of GBM, it is impossible to completely eradicate these malignant tumors though surgical resection. Inevitably, these tumors recur, thereby necessitating chemotherapy. TMZ is currently the 
A

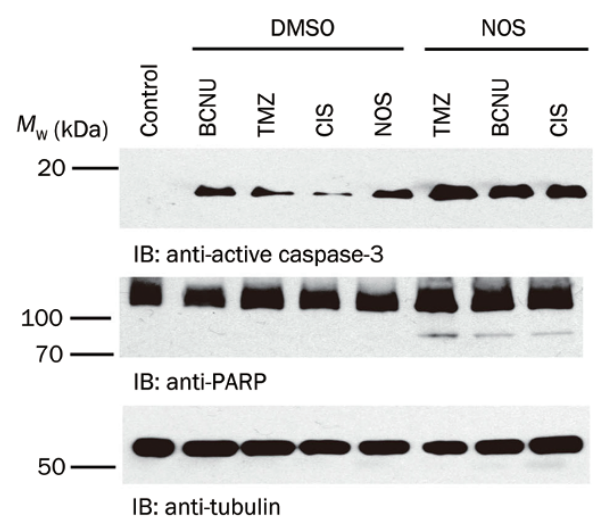

B

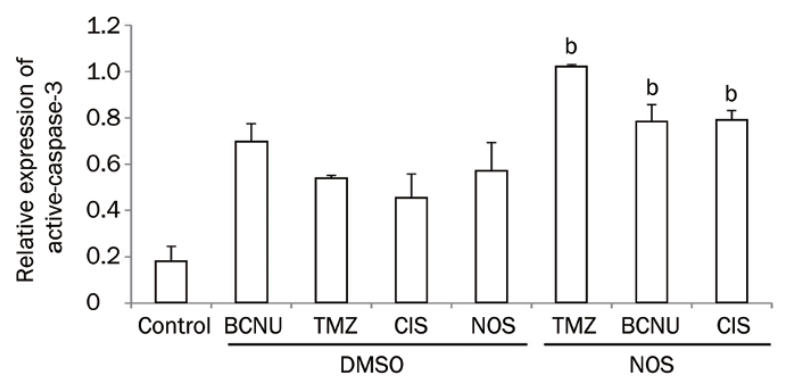

C

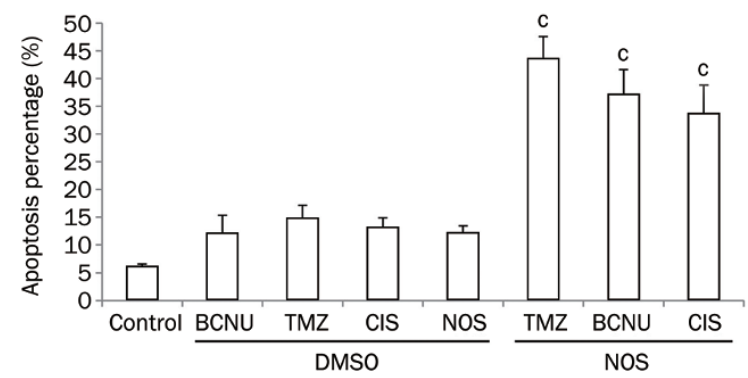

D

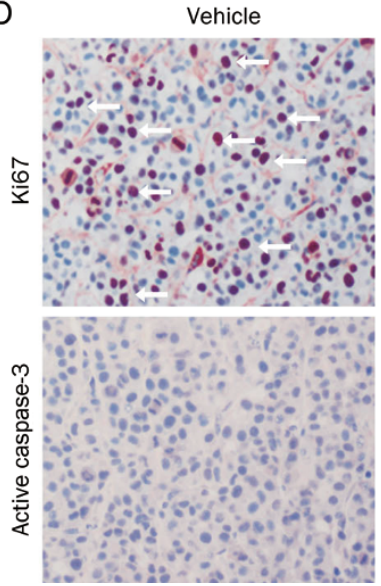

TMZ

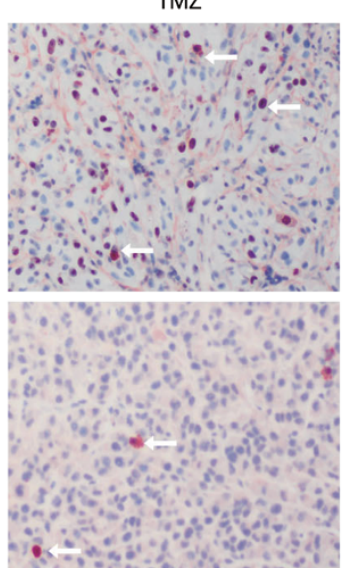

TMZ+NOS

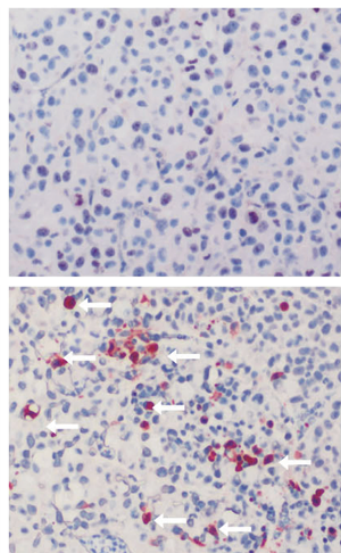

CIS

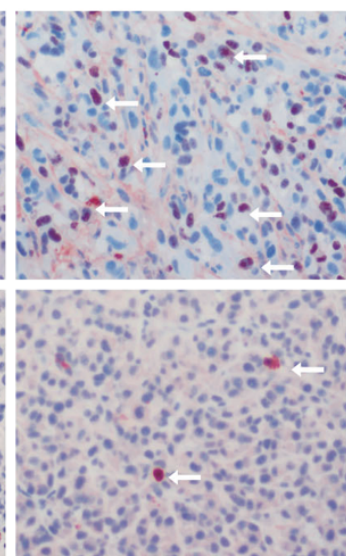

CIS+NOS

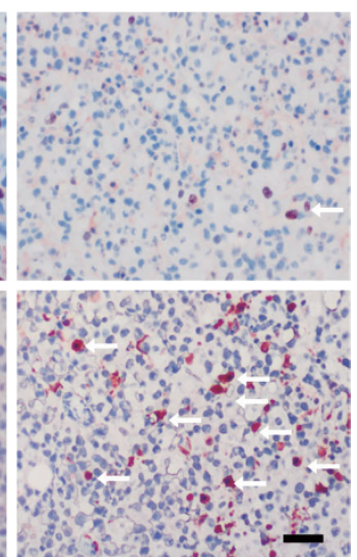

Figure 3. Combined treatment with chemotherapeutics and NOS enhances the expression of active-caspase-3. (A) Combination treatment enhanced active-caspase-3 expression in vitro. U87MG cells were treated with the indicated drugs and combinations (each at the concentration of $20 \mu \mathrm{mol} / \mathrm{L}$ ) for $72 \mathrm{~h}$. Cells were collected, and $50 \mu \mathrm{g}$ of total protein was used for Western blotting analysis with the indicated antibodies. (B) Active-caspase-3 expression was quantified as the expression ratio vs tubulin (Data represents the mean \pm SD. ${ }^{b} P<0.05$ compared to corresponding drugs respectively). (C) The synergistic effect of NOS and canonical chemotherapeutics on the induction of apoptosis in U87MG cells. Data represents the mean \pm SD. ${ }^{\mathrm{C}} P<0.01$ compared to corresponding drugs. (D) Combination treatment enhanced active-caspase-3 expression and decreased Ki67 expression in vivo. Immunohistochemical staining of active-caspase-3 and Ki67 in tumor sections derived from animals treated with indicated drugs. Brown represents the positively staining cells (arrows). Bar represents $100 \mu \mathrm{m}$. NOS, noscapine; TMZ, temozolamide; BCNU, bis-chloroethylnitrosourea; CIS, cisplatin.

standard of care for the treatment of GBM. To increase the anticancer potency of TMZ, a combinatorial strategy with other pharmacologic agents has previously been explored. For example, clinical trials have indicated that the addition of chloroquine might be beneficial for the treatment of glioma patients $^{[29]}$. In laboratory studies, it has been found that TMZ killed brain tumor cells more efficiently when epigallocatechin gallate (EGCG), a component of green tea, was added; however, the efficacy of this effect has not yet been confirmed in brain tumor patients ${ }^{[30]}$. Moreover, in clinical trials, the combination of BCNU $\left(150 \mathrm{mg} / \mathrm{m}^{2}\right)$ plus TMZ $\left(110 \mathrm{mg} / \mathrm{m}^{2}\right)$ as neo-adjuvant therapy in inoperable GBM exhibited promising activity with a good safety profile ${ }^{[31]}$, indicating that concomitant chemotherapy might be beneficial for patients with GBM.

Notably, it was previously shown that NOS and diltiazem could augment taxol- and radiation-induced S-phase arrest 

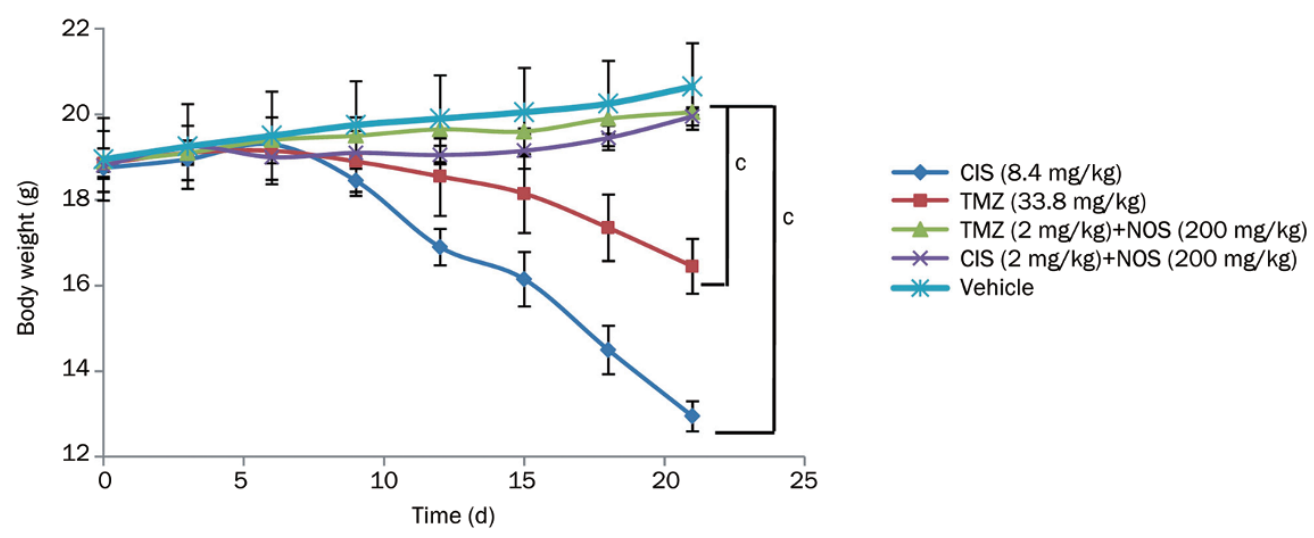

B
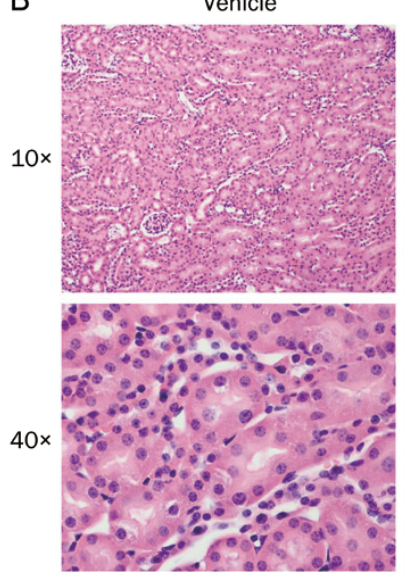

TMZ (33.8 mg/kg)
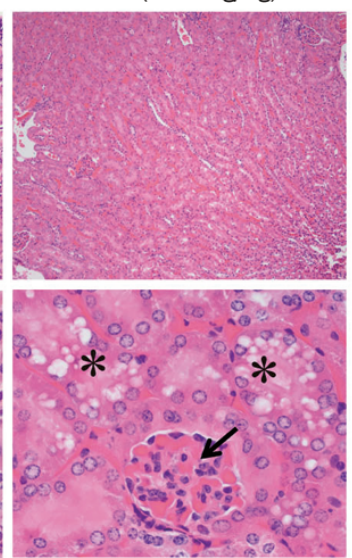

TMZ (2 mg/kg)+ NOS (200 mg/kg)
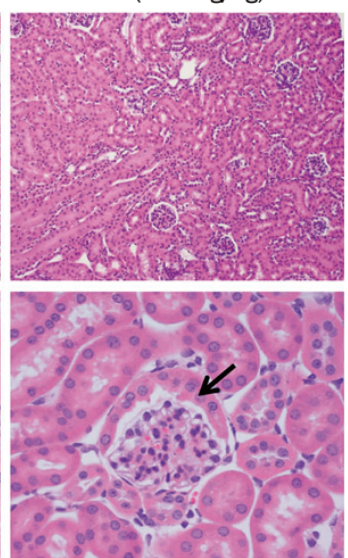

CIS (8.4 mg/kg)
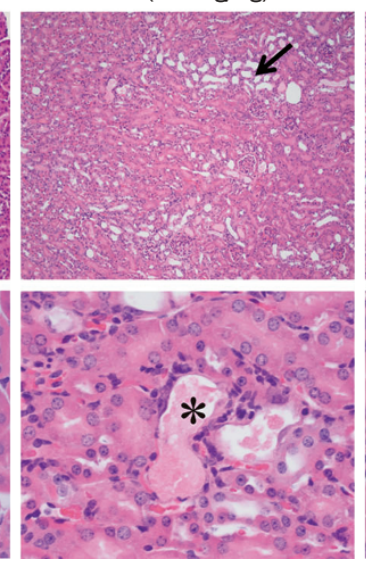

CIS $(2 \mathrm{mg} / \mathrm{kg})+$ $\operatorname{NOS}(200 \mathrm{mg} / \mathrm{kg})$

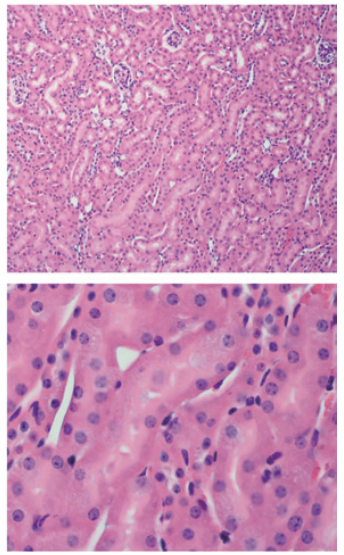

Figure 4. Toxicological evaluation in mice treated with NOS, TMZ, CIS, and the combination of NOS and TMZ or CIS. (A) Changes in the body weight of the mice in each respective treatment groups. Values represent the mean $\pm \mathrm{SD}(n=6) .{ }^{\mathrm{c}} P<0.01$ compared to vehicle controls. (B) Histological examination of the kidneys from different treatment groups. Compared to the vehicle, the kidneys from mice that received the combination of $\mathrm{CIS}$ ( 2 $\mathrm{mg} / \mathrm{kg}$ ) and NOS showed no significant histological changes. Kidneys from mice that received the combination of TMZ (2 mg/kg) and NOS exhibited subtle tubulization of the Bowman capsule (arrow). Kidneys from the mice that received high dose TMZ (33.8 mg/kg) showed significant peritubular and intraglomerular congestion (arrow) of the capillary vessels and focal coarse vacuolization of the proximal tubules (asterisk). Kidneys from the mice that received high dose CIS $(8.4 \mathrm{mg} / \mathrm{kg}$ ) showed mild congestion of the capillary vessels, focal acute tubular necrosis (asterisk) and dilation of the distal and proximal collection ducts (arrow). NOS, noscapine; TMZ, temozolamide; BCNU, bis-chloroethylnitrosourea; CIS, cisplatin.

and clonogenic death in C6 glioma cells in vitro ${ }^{[32]}$. In alignment with our exciting synergistic anti-cancer effect, exerted by NOS and various FDA-approved drugs, it was recently reported that NOS significantly decreases TMZ-resistant glioma cell growth and invasion. In the intracranial xenograft model, it was shown that NOS increases the survival of animals with TMZ-resistant gliomas. This study suggests that NOS can provide an alternative therapeutic approach for the treatment of TMZ-resistant gliomas ${ }^{[33]}$. NOS and its derivatives have been shown to be effective for a variety of other drug-resistant and hormone-refractory tumor types ${ }^{[4,16,34]}$.

FDA-approved cytotoxic anti-cancer drugs possess mechanism-based side effects and toxicities. For example, the most common non-hematological adverse effects associated with TMZ are nausea and vomiting. These effects are usually mild to moderate (grade 1 to 2 ). In addition, TMZ can have genotoxic effects. BCNU also has a variety of side effects, including pulmonary toxicity, hematologic toxicity, gastrointestinal toxicity, hepatotoxicity and renal abnormality. CIS has a number of side effects that can limit its use, of which nephrotoxicity is a major concern. At doses in the therapeutic range, CIS can also trigger neurotoxicity, nausea, vomiting, hearing loss and myelotoxicity. Our in vivo experiments show that when combined with NOS, a very low dose of these cytotoxic anti-cancer drugs can exert a potent tumor-inhibitive effect without organ toxicity. If this outcome holds true in the clinical setting, this approach will have a positive impact on both the quality of life of patients and the therapeutic outcome.

Our study shows that the combination of NOS and conventional therapeutics exhibits a synergistic effect against U87MG human glioblastoma cells. If NOS is used as an adjuvant in this manner, the dose of these approved anticancer drugs can be greatly reduced, reducing potential side effects and toxicities. The potential for combining NOS with conventional 
medicines as a chemotherapeutic strategy for the treatment of malignant tumors warrants further investigation.

\section{Acknowledgements}

This project was funded with federal funds from the National Cancer Institute, National Institutes of Health, R01 (CA127119 to Keqiang YE). We thank Obiamaka OBIANYO for proofreading the manuscript.

\section{Author contribution}

Qi QI and Keqiang YE designed the research; Qi QI and Xia LIU performed the experiments; Qi QI, Shiyong LI, Harish C JOSHI, and Keqiang YE analyzed the data; Qi QI and Keqiang $\mathrm{YE}$ wrote the paper.

\section{References}

1 Ye K, Ke Y, Keshava N, Shanks J, Kapp JA, Tekmal RR, et al. Opium alkaloid noscapine is an antitumor agent that arrests metaphase and induces apoptosis in dividing cells. Proc Natl Acad Sci U S A 1998; 95: 1601-6.

2 Landen JW, Lang R, McMahon SJ, Rusan NM, Yvon AM, Adams AW, et al. Noscapine alters microtubule dynamics in living cells and inhibits the progression of melanoma. Cancer Res 2002; 62: 4109-14.

3 Zhou J, Panda D, Landen JW, Wilson L, Joshi HC. Minor alteration of microtubule dynamics causes loss of tension across kinetochore pairs and activates the spindle checkpoint. J Biol Chem 2002; 277 : 17200-8.

4 Zhou J, Gupta K, Yao J, Ye K, Panda D, Giannakakou P, et al. Paclitaxel-resistant human ovarian cancer cells undergo c-Jun NH2terminal kinase-mediated apoptosis in response to noscapine. J Biol Chem 2002; 277: 39777-85.

5 Zhou J, Yao J, Joshi HC. Attachment and tension in the spindle assembly checkpoint. J Cell Sci 2002; 115: 3547-55.

6 Landen JW, Hau V, Wang M, Davis T, Ciliax B, Wainer BH, et al. Noscapine crosses the blood-brain barrier and inhibits glioblastoma growth. Clin Cancer Res 2004; 10: 5187-201.

7 Ke Y, Ye K, Grossniklaus HE, Archer DR, Joshi HC, Kapp JA. Noscapine inhibits tumor growth with little toxicity to normal tissues or inhibition of immune responses. Cancer Immunol Immunother 2000; 49: 217 25.

8 Empey DW, Laitinen LA, Young GA, Bye CE, Hughes DT. Comparison of the antitussive effects of codeine phosphate $20 \mathrm{mg}$, dextromethorphan $30 \mathrm{mg}$ and noscapine $30 \mathrm{mg}$ using citric acid-induced cough in normal subjects. Eur J Clin Pharmacol 1979; 16: 393-7.

9 Karlsson MO, Dahlstrom B, Eckernas SA, Johansson M, AIm AT. Pharmacokinetics of oral noscapine. Eur J Clin Pharmacol 1990; 39: 275-9.

10 Sammak PJ, Gorbsky GJ, Borisy GG. Microtubule dynamics in vivo: a test of mechanisms of turnover. J Cell Biol 1987; 104: 395-405.

11 Zhou J, Giannakakou P. Targeting microtubules for cancer chemotherapy. Curr Med Chem Anticancer Agents 2005; 5: 65-71.

12 Perez EA. Microtubule inhibitors: Differentiating tubulin-inhibiting agents based on mechanisms of action, clinical activity, and resistance. Mol Cancer Ther 2009; 8: 2086-95.

13 Ranganathan S, Dexter DW, Benetatos CA, Chapman AE, Tew KD, Hudes GR. Increase of beta(III)- and beta(IVa)-tubulin isotopes in human prostate carcinoma cells as a result of estramustine resistance. Cancer Res 1996; 56: 2584-9.

14 Giannakakou P, Sackett DL, Kang YK, Zhan Z, Buters JT, Fojo T, et al.
Paclitaxel-resistant human ovarian cancer cells have mutant betatubulins that exhibit impaired paclitaxel-driven polymerization. J Biol Chem 1997; 272: 17118-25.

15 Monzo M, Rosell R, Sanchez JJ, Lee JS, O’Brate A, Gonzalez-Larriba JL, et al. Paclitaxel resistance in non-small-cell lung cancer associated with beta-tubulin gene mutations. J Clin Oncol 1999; 17: 1786-93.

16 Aneja R, Vangapandu SN, Lopus M, Chandra R, Panda D, Joshi HC. Development of a novel nitro-derivative of noscapine for the potential treatment of drug-resistant ovarian cancer and T-cell lymphoma. Mol Pharmacol 2006; 69: 1801-9.

17 Sheleg SV, Korotkevich EA, Zhavrid EA, Muravskaya GV, Smeyanovich AF, Shanko YG, et al. Local chemotherapy with cisplatin-depot for glioblastoma multiforme. J Neurooncol 2002; 60: 53-9.

18 Datta K, Babbar P, Srivastava T, Sinha S, Chattopadhyay P. p53 dependent apoptosis in glioma cell lines in response to hydrogen peroxide induced oxidative stress. Int J Biochem Cell Biol 2002; 34: 148-57.

19 Chou TC, Motzer RJ, Tong Y, Bosl GJ. Computerized quantitation of synergism and antagonism of taxol, topotecan, and cisplatin against human teratocarcinoma cell growth: a rational approach to clinical protocol design. J Natl Cancer Inst 1994; 86: 1517-24.

20 Newcomb EW, Lukyanov Y, Schnee T, Ali MA, Lan L, Zagzag D. Noscapine inhibits hypoxia-mediated HIF-1alpha expression andangiogenesis in vitro: a novel function for an old drug. Int J Oncol 2006; 28: 1121-30.

21 Mahmoudian M, Rahimi-Moghaddam P. The anti-cancer activity of noscapine: a review. Recent Pat Anticancer Drug Discov 2009; 4: 92-7.

22 Chou TC. Theoretical basis, experimental design, and computerized simulation of synergism and antagonism in drug combination studies. Pharmacol Rev 2006; 58: 621-81.

23 Newcomb EW, Lukyanov Y, Smirnova I, Schnee T, Zagzag D. Noscapine induces apoptosis in human glioma cells by an apoptosisinducing factor-dependent pathway. Anticancer Drugs 2008; 19 : 553-63.

24 Antonyak MA, Kenyon LC, Godwin AK, James DC, Emlet DR, Okamoto I, et al. Elevated JNK activation contributes to the pathogenesis of human brain tumors. Oncogene 2002; 21: 5038-46.

25 Jeong M, Kwon YS, Park SH, Kim CY, Jeun SS, Song KW, et al. Possible novel therapy for malignant gliomas with secretable trimeric TRAIL. PloS One 2009; 4: e4545.

26 Kanzawa T, Germano IM, Kondo Y, Ito H, Kyo S, Kondo S. Inhibition of telomerase activity in malignant glioma cells correlates with their sensitivity to temozolomide. Br J Cancer 2003; 89: 922-9.

27 Brada M, Judson I, Beale P, Moore S, Reidenberg P, Statkevich P, et al. Phase I dose-escalation and pharmacokinetic study of temozolomide (SCH 52365) for refractory or relapsing malignancies. Br J Cancer 1999; 81: 1022-30.

28 Chen G, Pan QC. Potentiation of the antitumor activity of cisplatin in mice by 3-aminobenzamide and nicotinamide. Cancer Chemother Pharmacol 1988; 22: 303-7.

29 Gilbert MR. New treatments for malignant gliomas: careful evaluation and cautious optimism required. Ann Intern Med 2006; 144: 371-3.

30 Pyrko P, Schonthal AH, Hofman FM, Chen TC, Lee AS. The unfolded protein response regulator GRP78/BiP as a novel target for increasing chemosensitivity in malignant gliomas. Cancer Res 2007; 67: 980916.

31 Barrie M, Couprie C, Dufour H, Figarella-Branger D, Muracciole X, Hoang-Xuan $\mathrm{K}$, et al. Temozolomide in combination with BCNU before and after radiotherapy in patients with inoperable newly diagnosed glioblastoma multiforme. Ann Oncol 2005; 16: 1177-84. 
32 Altinoz MA, Bilir A, Del Maestro RF, Tuna S, Ozcan E, Gedikoglu G. Noscapine and diltiazem augment taxol and radiation-induced S-phase arrest and clonogenic death of 66 glioma in vitro. Surg Neurol 2006; 65: 478-84.

33 Jhaveri N, Cho H, Torres S, Wang W, Schonthal AH, Petasis NA, et al. Noscapine inhibits tumor growth in TMZ-resistant gliomas. Cancer
Lett 2011; 312: 245-52.

34 Aneja R, Miyagi T, Karna P, Ezell T, Shukla D, Vij Gupta M, et al. A novel microtubule-modulating agent induces mitochondrially driven caspase-dependent apoptosis via mitotic checkpoint activation in human prostate cancer cells. Eur J Cancer 2010; 46: 1668-78. 